\title{
Design of Radar Countermeasure Reconnaissance and Interference System
}

\author{
Ming LI \\ Ordnance Engineering College, Shijiazhuang 050003, P. R. China \\ liming_fly@126.com
}

\begin{abstract}
Keywords: radar confrontation, reconnaissance, interference
Abstract. The radar reconciliation and interference system is constructed, which is based on the function, composition, main technical indexes and implementation methods. The radar reconnaissance and jamming system is analyzed. The radar provides a theoretical basis for the prediction of the use and effect of reconnaissance and interference.
\end{abstract}

\section{Introduction}

Radar confrontation systems are usually more complex and costly, especially for some foreign radar confrontation tests, because there is no corresponding target radar entities involved in the test, making the test effectiveness is reduced. This paper constructs a radar reconnaissance reconnaissance and interference system. The system can simulate the typical radar, can complete the test radar against the system function and performance indicators of the test and verification, test the radar against the system hardware and software to adapt to the needs of multi-functional, but also for other platforms, electronic warfare system Provide field test means. Thus, it is possible to significantly shorten the test cycle of the radar countermeasure equipment, reduce the test cost and improve the test effectiveness.

\section{Radar counter reconnaissance and interference system function}

The main functions of the radar reconnaissance reconnaissance and interference system include: detecting the radar signal and measuring the radar signal parameters, processing the radar signal parameters, showing the radar reconnaissance results; the second is to generate the radar interference signal, the designated radar interference, Provide radar interference and anti-jamming practical training to interfere with the environment. The third is to provide radar in the process of training the target environment.

\section{System composition and working principle}

Radar counter reconnaissance and interference system structure design follows the principle of miniaturization, modularity, and structure. The system consists mainly of display control extension, radar reconnaissance reconnaissance host, reconnaissance / jamming antenna, as shown in Fig. 1.

The system works as follows: When the system is in the reconnaissance state, the received radar signal is controlled by the high power transmission and control switch, into the microwave combination, the limit magnification is divided into two passes through the power divider, one of which enters the instantaneous frequency measurement receiver, Frequency receiver to send the frequency code and signal time domain parameters, into the signal sorting module, the signal sorting module to send sorting results to the interference interface and control circuit, interference interface and control circuit through the network port to send data to the display control extension, Complete reconnaissance; another signal down the frequency of the IF signal into the digital radio frequency memory, the signal storage. When the system carries out radar interference, the display control extension sends the interference instruction, the interference interface and the control circuit, the instruction sent to the interference technology control module, the interference technology control module will control the digital radio frequency memory to complete the interference pattern 
generation according to the instruction, and the microwave combination, High-power transceiver switch control, the device issued an interference signal.

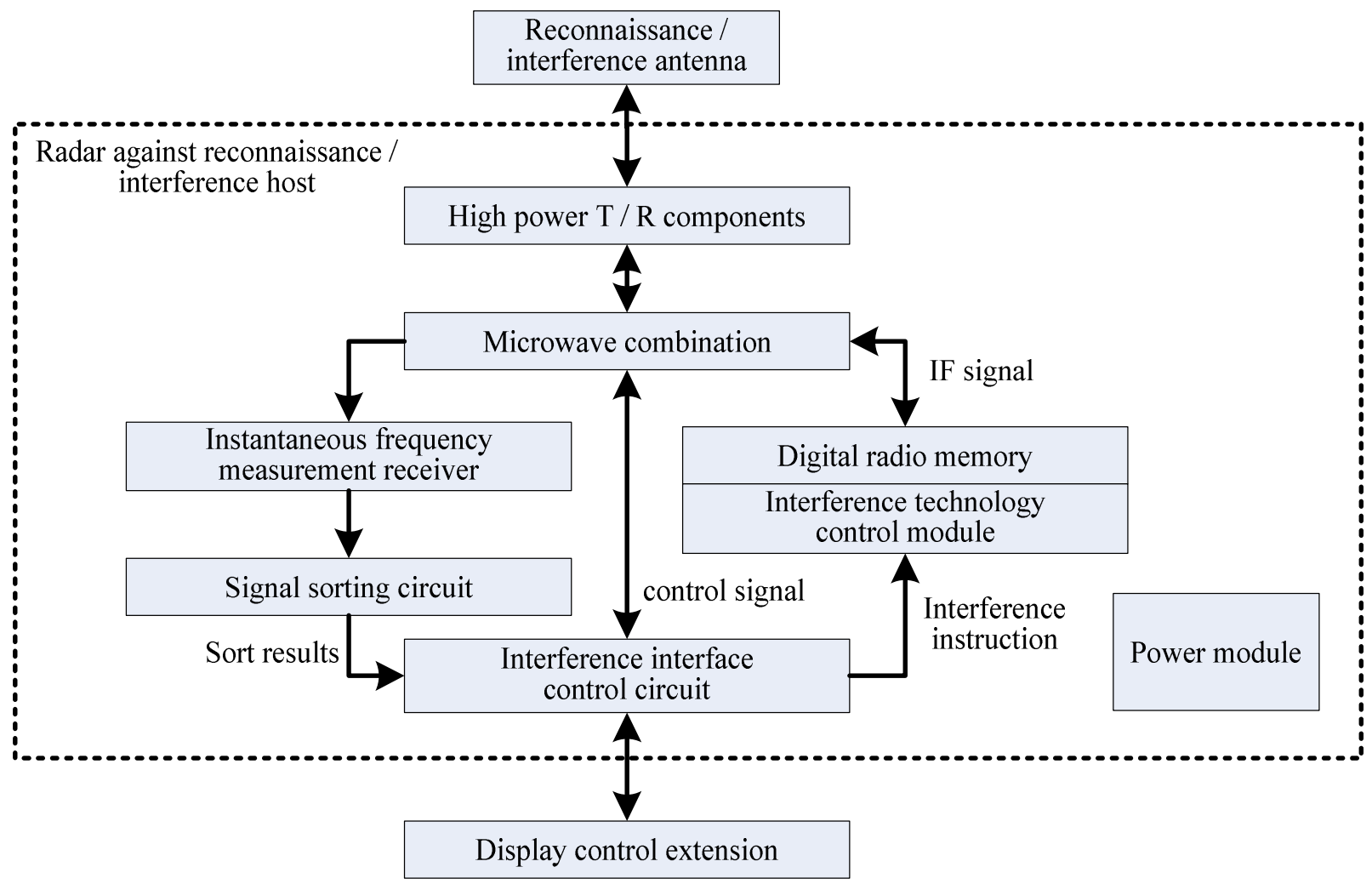

Fig.1 System block diagram

\section{System implementation}

(1) Display control extension

Display of the role of the control of the extension include: First, to display the system to detect the radar signal radiation source parameters (Including signal azimuth, carrier frequency, repetition rate, pulse width and other information), full pulse parameter information, the display system, and the display signal of the radar signal. Self-test and work status information, etc .; the second is based on reconnaissance results set interference parameters, control radar reconnaissance reconnaissance/interference host to produce different types of interference signals.

(2) Radar against reconnaissance/interference host

Radar reconnaissance reconnaissance/interference host mainly by the high-power T/R components, microwave combination, instantaneous frequency measurement receiver, signal sorting circuit, digital radio frequency memory, interference technology controller module, interference interface control circuit and power supply circuit composed of eight.

\section{1) Microwave combination}

Microwave combination is mainly used for RF signal up and down frequency control, the need to deal with the RF signal amplification, filtering, up and down frequency conversion. Including RF switch, low noise amplifier, limiting amplifier, filter, power splitter, coupler, attenuator, mixer, frequency synthesizer and so on.

2) Instantaneous frequency measurement receiver

Instantaneous frequency measurement receiver is mainly used to receive, measure the frequency of radar RF signals. The instantaneous frequency measurement receiver adopts the phase method to measure the frequency, mainly composed of the limiting magnification filter module, the directional coupler, the single pole single throw switch, the detector, the eight power divider, the delay line, the 
phase detector and the quantization coding circuit. Wherein the phase detector is adapted to compare the phase difference between the input delayed and non-delayed signals; the quantization coding circuit encodes the phase information of each channel phase detector and finally outputs the frequency code.

\section{3) Signal sorting circuit}

The main function of the signal sorting circuit is to achieve the radar pulse time domain parameters (pulse width, pulse amplitude, pulse arrival time) measurement, the formation of pulse description word, and pulse description word signal sorting and other processing work, The source file. Signal processing system mainly by the signal processing circuit and VIM6413 processing circuit and other components. Among them, the main signal processing circuit is to achieve the radar signal pulse time domain parameters of the measurement, and the formation of pulse description word; VIM6413 processing circuit is the role of the signal from the main processing circuit to read the pulse description word, the signal sorting.

4) Digital storage frequency

The role of digital storage frequency, including two: First, the front of the IF signal sent digital, and storage; Second, according to the interference control command to generate radar interference signal.

Digital storage frequency is mainly composed of three parts: instantaneous bandwidth digital memory, interference technology generator and digital noise generator. Wherein the instantaneous bandwidth digital memory is used to digitize and store the input intermediate frequency signal; when the signal needs to be restored, the intermediate frequency signal is restored and input. The effect of the interference technology generator is to receive the interference control command from the host computer to generate the interference signal that meets the requirements. Digital noise generator is the role of the reference signal through the frequency shift and noise modulation, as a mixed signal.

Digital frequency control work process: interference technology generator to receive external control signals (mainly transient output signal), or according to the internal preset interference parameters, guide the frequency synthesizer to switch to the appropriate down-conversion local oscillator, so down-conversion Component output frequency in the medium frequency range, the digital RF memory input signal sent to the instantaneous bandwidth of digital memory for storage, interference technology generator according to interference mode control instantaneous bandwidth digital memory for signal reduction, and according to the interference mode, control the output signal plus the Modulation, including Doppler shift, narrowband noise modulation, the formation of interference modulation signal, control the frequency synthesizer to switch to the appropriate up-conversion local oscillator, the interference modulation signal up-conversion mixing, complete the input signal recovery process The

\section{Conclusions}

Based on the reality of radar confrontation system, this paper expounds the function of radar reconnaissance and jamming system, explains the composition and working principle of the system, and gives the working mode and working flow of the system. The radar reconnaissance and interference system can simulate all kinds of radar, easy to use, easy to operate. Through the preset parameter template design, a variety of typical radar can be set up a key, can be convenient and effective to complete the test radar against the system function and performance indicators of testing and verification.

\section{References}

[1] Wang Jian. Radar semi-physical simulation system design and implementation [D]. Xi'an: Xi'an University of Electronic Science and Technology, 2009: 1-10. 
[2] Xu Hailong, Zhou Shuitou, Li Hongzhong. Application and effectiveness analysis of active bait combat for shipboard outboard radar $[\mathrm{J}]$. Electronic Information Countermeasure Technology, 2007,22 (4): 50-53.

[3] Hu Hang, Deng Xinhong. The sidelobe suppression method of sub - array planar phased array ADBF [J]. Journal of Radio Science, 2008,23 (1): 201-205.

[4] Zeng Cao, He Xuehui, Liao Guisheng, and so on. Multi - beam multi - beam forming method for symmetric exponential distribution [J]. Systems Engineering and Electronics, 2009,31 (10): 2294-2295.

[5] Zhang Qiang. Electronic warfare semi-physical simulation system design [J]. Ship Electronic Engineering, 2006,26 (4): 111-114.

[6] Liu Jiaqi, Zhou Yan, Wang Guoyu, etc. Radar electronic warfare semi-physical simulation system [J]. Missile and space carrying technology, 2006 (6): 29-32.

[7] Dan Mei, Wang Xuesong, clear, etc. Radar electronic warfare semi-physical simulation system in the database technology [J]. Space electronic warfare, 2002 (2): 8-11. 